\title{
Fixed-Point Theorem for Isometric Self-Mappings
}

\author{
Joseph Frank Gordon (iD) \\ Department of Mathematics, Nanjing University of Aeronautics and Astronautics, Nanjing 211106, China \\ Correspondence should be addressed to Joseph Frank Gordon; jgordon@aims.edu.gh
}

Received 6 July 2020; Revised 21 September 2020; Accepted 24 September 2020; Published 13 October 2020

Academic Editor: Jean Michel Rakotoson

Copyright () 2020 Joseph Frank Gordon. This is an open access article distributed under the Creative Commons Attribution License, which permits unrestricted use, distribution, and reproduction in any medium, provided the original work is properly cited.

In this paper, we derive a fixed-point theorem for self-mappings. That is, it is shown that every isometric self-mapping on a weakly compact convex subset of a strictly convex Banach space has a fixed point.

\section{Introduction}

Let $\mathscr{X}$ be a Banach space and $\mathscr{C}$ be a closed convex subset $\mathscr{X}$. Let $T: \mathscr{C} \longrightarrow \mathscr{C}$ be a self-mapping of $\mathscr{C}$. Recall that $T$ is said to be nonexpansive if

$$
\|T x-T y\| \leq\|x-y\|,
$$

for all $x, y \in \mathscr{C}$. The fixed-point set of $T$ is $\operatorname{Fix}(T):=\{x \in \mathscr{C}: T x=x\}$. We say that the subset $\mathscr{C}$ of $\mathscr{X}$ is said to have an approximate fixed-point sequence for a nonexpansive self-map $T$ if

$$
\lim _{n \longrightarrow \infty}\left\|x_{n}-T x_{n}\right\|=0,
$$

for any sequence $\left\{x_{n}\right\}_{n \geq 1} \subset \mathscr{C}$. When the closed convex subset $\mathscr{C}$ is bounded, then such a sequence always exists; indeed, by letting $\varepsilon_{n} \in(0,1)$, for all $n \geq 1$, be a null sequence and defining the maps $T_{n} x=\varepsilon_{n} x_{0}+\left(1-\varepsilon_{n}\right) T x$ where arbitrarily $x_{0} \in \mathscr{C}$, one can see that $\left\|T_{n} y-T_{n} x\right\| \leq \quad\left(1-\varepsilon_{n}\right) \| T y-$ $T x\left\|\leq\left(1-\varepsilon_{n}\right)\right\| y-x \|$, implying that $T_{n}$ is a contraction mapping with contraction constant $1-\varepsilon_{n}$. By the Banach contraction mapping theorem, it follows that there exists a unique $x_{n} \in \mathscr{C}$ such that $T_{n} x_{n}=x_{n}$, which implies that

$$
x_{n}=\varepsilon_{n} x_{0}+\left(1-\varepsilon_{n}\right) T x_{n},
$$

from which we get $\left\|x_{n}-T x_{n}\right\|=\varepsilon_{n}\left\|x_{0}-T x_{n}\right\|$ $\leq \varepsilon_{n} \sup _{x, y \in \mathscr{C}}\|x-y\|$. Given that $\varepsilon_{n} \longrightarrow 0$ and $\sup _{x, y \in \mathscr{C}}\|x-y\|<\infty$, it follows that $\left\{x_{n}\right\}_{n \geq 1}$ is an approximate fixed-point sequence.
Another way of constructing an approximate fixed-point sequence is to require or assume that $\operatorname{Fix}(T)$ is nonempty. Now due to the assumption that $\operatorname{Fix}(T) \neq \varnothing$, the sequence $\left\{x_{n}\right\}_{n \geq 1} \subset \mathscr{C}$ is bounded (indeed, $\left\|x_{n}-p\right\| \leq\left\|x_{0}-p\right\|$ for all $p \in \operatorname{Fix}(T)$ and $x_{0}$ taken arbitrarily in $\mathscr{C}$ ). Hence,

$$
\left\|x_{n}-T x_{n}\right\|=\varepsilon_{n}\left\|x_{0}-T x_{n}\right\| \longrightarrow 0
$$

and $\left\{x_{n}\right\}_{n \geq 1}$ is an approximating fixed point for $T$.

A mapping $T: \mathscr{C} \longrightarrow \mathscr{C}$ of a set $\mathscr{C}$ in a Banach space $\mathscr{X}$ is called isometric if

$$
\|T x-T y\|=\|x-y\|,
$$

for all $x, y \in \mathscr{C}$. Note that an isometric mapping is just a nonexpansive mapping in which the inequality is always an equality. A well-known result of Brodskii and Milman [1] asserts that if $\mathscr{C}$ is a weakly compact convex subset of $\mathscr{X}$ and $\mathscr{X}$ has normal structure, then $\mathscr{X}$ has the fixed-point property for isometric mappings. In particular, any compact convex subset of $\mathscr{X}$ has the fixed-point property (see [2]).

References [3-10] can be consulted for fixed-point problems on isometric mappings.

Definition 1 (strictly convex Banach space). A strictly convex Banach space is a Banach space such that whenever $x \neq 0 \neq y$, then $\|x+y\|=\|x\|+\|y\|$ if and only if $x=\lambda y$ for some $\lambda>0$.

An example of a strictly convex Banach space is a Hilbert space. 
Definition 2 (convex linear). Let $\mathscr{X}$ be a Banach space and $\mathscr{C} \subseteq \mathscr{X}$ be a closed convex subset of $\mathscr{X}$. Then, the map $T: \mathscr{C} \longrightarrow \mathscr{X}$ is said to be a convex linear if

$$
T(a x+(1-a) y)=a T x+(1-a) y,
$$

for all $x, y \in \mathscr{C}$ and $a \in(0,1)$.

An example of a convex linear is a linear map.

\section{Preliminaries}

We introduce the following useful theorems that will be used in the proof of our main result.

Theorem 1 (Mazur's theorem). Every nonempty convex subset of a Banach space is strongly closed if and only if it is weakly closed.

Theorem 2 (Cantor's intersection theorem). Let $\mathscr{X}$ be $a$ topological space. A decreasing nested sequence of nonempty compact closed subset of $\mathscr{X}$ has nonempty intersection. In other words, suppose that $\mathscr{C}_{k}$ is a sequence of nonempty compact closed subset of $\mathscr{X}$ satisfying $\mathscr{C}_{0} \mathscr{C}_{1} \ldots . . \mathscr{C}_{n}^{\mathscr{C}_{n+1} \cdots}$, and it follows that

$$
\left(\bigcap_{k} \mathscr{C}_{k}\right) \neq \varnothing
$$

\section{Main Result}

We give the proof of the main result of this paper, which is accomplished in Theorem 3 below. The following lemma, corollary, and proposition shall aid us in arriving at the conclusion of the main result.

Lemma 1. Let $\mathscr{X}$ be a strictly convex Banach space and $\mathscr{C} \subseteq \mathscr{X}$ be a closed convex subset and $T: \mathscr{C} \longrightarrow \mathscr{C}$ be an isometric mapping. Then, $T$ is convex linear on $\mathscr{C}$. That is, $T(a x+$ $(1-a) y)=a T x+(1-a) T y$ for all $x, y \in \mathscr{C}$ and $a \in(0,1)$.

Proof. Let $w=a x+(1-a) y$ and $a \in(0,1)$. Without loss of generality, assume $x \neq y$. Then, $w-x=(1-a)(y-x)$ implies that

$$
\|w-x\|=(1-a)\|y-x\|
$$

Similarly, $w-y=a(x-y)$ which also implies that

$$
\|w-y\|=a\|x-y\| .
$$

First, we show that $T x \neq T y \neq T w$. To see this, we observe that if $T x=T w$, then from (9), we have

$$
\begin{aligned}
\|w-y\| & =a\|x-y\| \\
& =a\|T x-T y\| \\
& =a\|T w-T y\| \\
& =a\|w-y\|,
\end{aligned}
$$

leading to the contradiction that $a=1$.

Similarly, if $T y=T w$, then from (8), we have

$$
\begin{aligned}
\|w-x\| & =(1-a)\|y-x\| \\
& =(1-a)\|T y-T x\| \\
& =(1-a)\|T w-T x\| \\
& =(1-a)\|w-x\|,
\end{aligned}
$$

leading to the contradiction that $a=0$.

Now since $T$ is an isometry, it follows that

$$
\begin{aligned}
\|T x-T y\| & =\|T x-T w+T w-T y\| \\
& \leq\|T x-T w\|+\|T w-T y\| \\
& =\|x-w\|+\|w-y\| \\
& =(1-a)\|x-y\|+a\|x-y\| \\
& =\|x-y\| \\
& =\|T x-T y\|,
\end{aligned}
$$

which implies that $\|T x-T w\|+\|T w-T y\|=\|T x-T y\|$. Since $\mathscr{X}$ is strictly convex and $T x \neq T w \neq T y$ implies that there exist $\lambda>0$ such that

$$
\begin{aligned}
T x-T w & =\lambda(T w-T y), \\
(1+\lambda) T w & =T x+\lambda T y \\
T w & =\frac{1}{1+\lambda} T x+\frac{\lambda}{1+\lambda} T y,
\end{aligned}
$$

we obtain

$$
T w=\beta T x+(1-\beta) T y,
$$

where $\beta \in(0,1)$ and $\beta=1 /(1+\lambda)$. We finally show that $\beta=a$.

From (14), we have $T w-T x=(1-\beta)(T y-T x)$ which implies that

$$
\|T w-T x\|=(1-\beta)\|T y-T x\| .
$$

Also, $T w-T y=\beta(T x-T y)$ implies that

$$
\|T w-T y\|=\beta\|T x-T y\| .
$$

From (16), $\beta\|T x-T y\|=\|T w-T y\|=\|w-y\|=a \| x-$ $y\|=a\| T x-T y \|$ which implies that $\beta=a$. Hence, from (14), we have shown that $T(a x+(1-a) y)=a T x+(1-$ a) $T y$ which establishes the convex linearity of $T$.

Corollary 1. Let $\mathscr{X}$ be a strictly convex Banach space, $\mathscr{C} \subseteq \mathscr{X}$ be a closed convex subset, and $T: \mathscr{C} \longrightarrow \mathscr{C}$ be an isometric mapping. Then, the function $f: \mathscr{C} \longrightarrow \mathbb{R}, x \longrightarrow$ $\|(I-T) x\|$ is a continuous convex function.

Proof. From Lemma 1, $T$ is a convex linear. Hence, subtracting the term $a x+(1-a) y$ from both sides of $T(a x+$ $(1-a) y)=a T x+(1-a) T y$ for all $x, y \in \mathscr{C}$ and $a \in(0,1)$, we have 


$$
(I-T)(a x+(1-a) y)=a(I-T) x+(1-a)(I-T) y .
$$

We have the following evaluation:

$$
\begin{aligned}
f(a x+(1-a) y) & =\|(1-T)(a x+(1-a) y)\| \\
& =\|a(1-T) x+(1-a)(1-T) y\| \\
& \leq a\|(1-T) x\|+(1-a)\|(1-T) y\| \\
& =a f(x)+(1-a) f(y) .
\end{aligned}
$$

Thus, $f$ is a convex function and continuous (because $T$ is continuous).

Proposition 1. Let $f: C \longrightarrow \mathbb{R}$ be continuous convex function on a weakly compact convex subset $\mathscr{C}$ of any Banach space $\mathscr{X}$. Then, $f$ attains its minimum on $\mathscr{C}$. That is, there exist $\bar{x} \in \mathscr{C}$ such that

$$
f(\bar{x})=\min \{f(x): x \in \mathscr{C}\} .
$$

Proof. Let $m=\inf \{f(x): x \in \mathscr{C}\}$. We show that $m>-\infty$ and that $f(\bar{x})=m$ for some $\bar{x} \in \mathscr{C}$.

Suppose that $m=-\infty$, and for each $n \in \mathbb{N}$, define $\mathscr{C}_{n}=\{x \in \mathscr{C}: f(x) \leq-n\}$. For each $n \in \mathbb{N}$, the set $\mathscr{C}_{n}$ is closed (and weakly closed by Theorem 1), convex, and nonempty (since $m=-\infty$ ). Therefore, $\left(\mathscr{C}_{n}\right)_{n=1}^{\infty}$ forms a nested decreasing sequence of weakly compact nonempty sets. By Theorem $2, \cap_{n=1}^{\infty} \mathscr{C}_{n} \neq \varnothing$. But this implies that there is some $\bar{x} \in \mathscr{C}$ such that $f(\bar{x}) \leq-n$ for all $n \in \mathbb{N}$, an impossibility. Consequently, $m>-\infty$.

So define $n \in \mathbb{N}$ a sequence of sets $\mathscr{C}_{n}^{\prime}=\{x \in \mathscr{C}: f(x) \leq m+(1 / n)\}$ for all $n$ belonging to $\mathrm{N}$ (natural numbers). As before, $\left(\mathscr{C}_{n}^{\prime}\right)_{n=1}^{\infty}$ is a nested sequence of weakly compact nonempty sets and so $\cap_{n=1}^{\infty} \mathscr{C}_{n}^{\prime} \neq \varnothing$. If $\bar{x} \in \cap_{n=1}^{\infty} \mathscr{C}_{n}^{\prime}$, then $\bar{x} \in \mathscr{C}$ and $f(\bar{x})=m$ as required.

Theorem 3 (main result). Every isometric self-mapping $T: \mathscr{C} \longrightarrow \mathscr{C}$ on a weakly compact convex subset $\mathscr{C}$ of a strictly convex Banach Space $\mathscr{X}$ has a fixed point.

Proof. We know from Corollary 1 that $f(x)$ : $=\|(I-T) x\|$ is a continuous convex function. So, by Proposition $1, f$ attains its minimum on $C$, say $\bar{x}$. By the approximate fixed point of $T$, it is always possible to choose a sequence $\left\{x_{n}\right\}_{n \geq 1} \subset \mathscr{C}$ such that $f\left(x_{n}\right)=\left\|x_{n}-T x_{n}\right\| \leq(1 / n)$ for all $n \geq 1$. Hence, $\|(I-T) \bar{x}\|=\inf _{x \in \mathscr{C}}\|(I-T) x\|=0$ and $\quad$ so
$T \bar{x}=\bar{x}$.

Example 1. Let $\mathscr{C}=[0,1]$ and $T:[0,1] \longrightarrow[0,1], T x=1-$ $x$ for all $x \in[0,1]$. Then, $T$ is an isometry, and by Theorem 3 , $T$ has a fixed point.

As an application of Theorem 3, it is desired to solve the linear problem

$$
x-v=\lambda(T x-v), \quad \lambda \in(0,1),
$$

for some nonzero scalar $\lambda$ and constant vector $v$, where $T: \mathscr{C} \longrightarrow \mathscr{C}$ is linear and $\mathscr{C}$ is closed and convex. The following theorem gives a solution.

Theorem 4. Let $\mathscr{C}$ be a closed convex subset of a Banach space $\mathscr{X}$ and let $T: \mathscr{C} \longrightarrow \mathscr{C}$ be linear and continuous. Then, equation (20) has a solution if the following holds:

(1) $\|T\|_{B(x, x)}=\lambda^{-1}$

(2) $\mathscr{C}$ is weakly compact

(3) $\mathscr{X}$ is strictly convex

Proof. By defining the auxiliary mapping

$$
T^{\prime}: \mathscr{C} \longrightarrow \mathscr{C}, x \longmapsto \lambda T x+(1-\lambda) v,
$$

it is clearly seen that $T^{\prime}$ is well defined and invariant on $\mathscr{C}$ since $\mathscr{C}$ is convex and $\lambda \in(0,1)$. Since $T^{\prime} y-T^{\prime} x=\lambda(T y-T x)$, then by taking norms gives us

$$
\left\|T^{\prime} y-T^{\prime} x\right\|_{x}=\lambda\|T y-T x\|_{x} .
$$

Since $T$ is bounded, then

$$
\begin{aligned}
\left\|T^{\prime} y-T^{\prime} x\right\|_{\mathscr{X}} & \leq \lambda\|T\|_{B(\mathscr{X}, \mathscr{X})}\|y-x\|_{\mathscr{X}}=\lambda \cdot \lambda^{-1}\|y-x\|_{\mathscr{X}} \\
& =\|y-x\|_{\mathscr{X}} .
\end{aligned}
$$

Since $T^{\prime}$ is an isometry, then by Theorem 3 , one can find a solution $\bar{x}=T^{\prime} \bar{x}=\lambda T \bar{x}+(1-\lambda) v$.

\section{Data Availability}

No data were used to support this study.

\section{Conflicts of Interest}

The author declares that there are no conflicts of interest.

\section{References}

[1] M. S. Brodskii and D. P. Milman, "On the center of a convex set," Doklady Akademii Nauk SSSR, vol. 59, pp. 837-840, 1948.

[2] DeM. Ralph, "Common fixed points for commuting contraction mappings," Pacific Journal of Mathematics, vol. 13, no. 4, pp. 1139-1141, 1963

[3] A. T. M. Lau, "Sets with fixed point property for isometric mappings," Proceedings of the American Mathematical Society, vol. 79, no. 3, p. 388, 1980.

[4] S. Rajesh and P. Veeramani, "Lim's center and fixed-point theorems for isometry mappings," Annals of Functional Analysis, vol. 9, no. 2, pp. 190-201, 2018.

[5] M. R. Themistocles, "Properties of isometric mappings," Journal of Mathematical Analysis and Applications, vol. 235, no. 1, pp. 108-121, 1999.

[6] D. G. Bourgin, "Classes of transformations and bordering transformations," Bulletin of the American Mathematical Society, vol. 57, no. 4, pp. 223-238, 1951.

[7] J. A. Baker, "Isometries in normed spaces," The American Mathematical Monthly, vol. 78, no. 6, pp. 655-658, 1971. 
[8] D. Richard, "Bourgin, Approximate isometries on finite dimensional banach spaces," Transactions of the American Mathematical Society, vol. 207, pp. 309-328, 1975.

[9] K. Ciesielski and T. M. Rassias, "On some properties of isometric mappings," Facta Universitatis, Series: Mathematics and Informatics, vol. 2, pp. 49-52, 1992.

[10] J. Fickett, "Approximate isometries on bounded sets with an application to measure theory," Studia Mathematica, vol. 72, no. 1, pp. 37-46, 1982. 